\title{
The Solution of Integral Equations in Chebyshev Series
}

\author{
By R. E. Scraton
}

\begin{abstract}
If the solution of an integral equation can be expanded in the form of a Chebyshev series, the equation can be transformed into an infinite set of algebraic equations in which the unknowns are the coefficients of the Chebyshev series. The algebraic equations are solved by standard iterative procedures, in which it is not necessary to determine beforehand how many coefficients are significant. The method is applicable to equations of either Fredholm or Volterra types.
\end{abstract}

Introduction. The solution of integral equations in Chebyshev series has been the subject of two papers by Elliott [1], [2]. Elliott's method is essentially a collocation method, and it is necessary to decide in advance how many terms in the Chebyshev series are likely to be significant. The method suggested here avoids this difficulty; in many respects it is similar to the method for differential equations given in an earlier paper by the present author [3].

Cons:der first the Fredholm equation

$$
y(x)=F(x)+\lambda \int_{-1}^{1} K(x, \xi) y(\xi) d \xi .
$$

It is assumed that the variables have been suitably transformed so as to reduce the range of integration to $(-1,1)$.

Let

$$
y(x)=\sum_{r=0}^{\infty} a_{r} T_{r}(x)
$$

and

$$
F(x)=\sum_{r=0}^{\infty} f_{r} T_{r}(x),
$$

where, in the usual way, $\sum^{\prime}$ denotes a sum whose first term is halved. If, then, $b_{r s}$ is defined by

$$
\sum_{r=0}^{\infty} b_{r s} T_{r}(x)=\int_{-1}^{1} K(x, \xi) T_{s}(\xi) d \xi
$$

it is easily seen that

$$
a_{r}-\lambda \sum_{s=0}^{\infty} b_{r s} a_{s}=f_{r}, \quad r=0,1,2, \cdots .
$$

The solution of the integral equation may now be divided into two parts: first, the evaluation of the coefficients $b_{r s}$; and, secondly, the solution of the algebraic Eq. (2).

Received October 27, 1966, revised January 31, 1969. 
Evaluation of the Coefficients $b_{r s}$. It will be assumed that the kernel $K(x, \xi)$ can be expanded in a double Chebyshev series

$$
K(x, \xi)=\sum_{r=0}^{\infty} \sum_{s=0}^{\infty} k_{r s} T_{r}(x) T_{s}(\xi) .
$$

This implies that the kernel must be bounded throughout the region $-1 \leqq x \leqq 1$, $-1 \leqq \xi \leqq 1$. From the properties of Chebyshev polynomials, it can be seen that

$$
\begin{aligned}
b_{r s}= & k_{r s}-\frac{1}{1 \cdot 3}\left(k_{r, 8+2}+\left.k_{r, \mid}\right|_{8-2} \mid\right)-\frac{1}{3 \cdot 5}\left(k_{r, s+4}+k_{r, \mid s-4} \mid\right) \\
& -\frac{1}{5 \cdot 7}\left(k_{r, s+6}+k_{r, \mid s-6}\right) \cdots
\end{aligned}
$$

so that $b_{r s}$ can be obtained immediately if the coefficients in the series (3) are known. It may happen that the series (3) is already known, or can be obtained in terms of other known series, and in such cases there is no further problem. In other cases, the coefficients $k_{r s}$ can be obtained by an obvious extension of the usual harmonic analysis formulae; that is, if

$$
S_{i s}=\sum_{j=0}^{n}{ }^{\prime \prime} K\left(\cos \frac{i \pi}{m}, \cos \frac{j \pi}{n}\right) \cos \frac{s j \pi}{n},
$$

then

$$
\frac{1}{4} m n k_{r s}=\sum_{i=0}^{m}{ }^{\prime \prime} S_{i s} \cos \frac{r i \pi}{m},
$$

provided that $m$ and $n$ are sufficiently large for $k_{r s}$ to be negligible if $r \geqq m$ or $s \geqq n$. (As usual, $\sum$ " denotes a sum whose first and last terms are halved.)

It should be mentioned at this stage that if the $k_{r s}$ are evaluated by using Eqs. (5) and (6), this evaluation accounts for a substantial part of the total computing time. Moreover, the choice of suitable values for $m$ and $n$ introduces some of the disadvantages of the collocation method. The greatest value of the present method therefore lies in those problems where the series (3) can be found without resorting to harmonic analysis; but even in other problems the method appears to compare favourably with the collocation method.

Solution of the Algebraic Equations. When the $b_{r s}$ have been obtained, the problem reduces to the solution of the infinite set of Eqs. (2). An approximate solution can always be obtained by solving the first $N$ of these equations for the first $N$ unknowns; but the choice of a suitable value for $N$ must necessarily be somewhat arbitrary. Instead, the iterative procedures given below may be used; these are similar to the procedures for differential equations given in the author's earlier paper [3].

First consider the problem where $F(x)$ is not identically zero and where $\lambda$ is given. The set of Eqs. (2) is then an infinite set of linear simultaneous equations. Now a study of Eq. (4) shows that the largest term on the right-hand side is likely to be the one which includes $k_{r 0}$ or $k_{r 1}$, and this term has a multiplier of order $1 / s^{2}$. Since $k_{r 0}$ and $k_{r 1}$ can be expected to decrease rapidly as $r$ increases, it follows that 
$b_{r s}$ is likely to be small unless $r$ and $s$ are both small. Provided that $\lambda$ is not too large, therefore, the majority of the off-diagonal terms in (2) may be expected to be small compared with the diagonal terms, and this suggests a Gauss-Seidel method of solution. The precise conditions required for convergence will be discussed later. The appropriate iterative formula is given by

$$
a_{r}{ }^{(k)}=\frac{1}{1-\lambda b_{r r}}\left[f_{r}+\lambda\left(\sum_{s=0}^{r-1} b_{r s} a_{s}{ }^{(k)}+\sum_{s=r+1}^{\infty} b_{r s} a_{s}{ }^{(k-1)}\right)\right],
$$

where, in the case $r=0$, the denominator $1-\lambda b_{r r}$ must be replaced by $1-\frac{1}{2} \lambda b_{00}$. The calculation proceeds in the obvious way, as many of the $a_{r}$ 's as are significant being retained at each stage of the iteration. The initial values $a_{r}{ }^{\left({ }^{0}\right)}$ may be taken as zero.

Secondly, consider the eigenvalue problem where $F(x)$ vanishes identically and $\lambda$ is unknown. The set of Eqs. (2) can then be written as

$$
\sum_{s=0}^{\infty} b_{r s}^{\prime} a_{s}=\Lambda a_{r},
$$

where $\Lambda=1 / \lambda$ is a latent root of the infinite matrix $\left[b_{r s}\right]$. The dominant value of $\Lambda$ (corresponding to the smallest eigenvalue $\lambda$ ) can be found by iteration in the usual way.

The usual practice would be to normalise the characteristic function so that the largest of the coefficients $a_{r}$ is equal to unity. In many cases $a_{0}$ is the largest coefficient, or at least is of the same order of magnitude as the largest coefficient, and it is convenient to normalise so that $a_{0}=1$, giving the iterative formulae:

$$
\begin{aligned}
& \Lambda^{(k)}=\frac{1}{2} b_{00}+\sum_{s=1}^{\infty} b_{0 s} a_{s}{ }^{(k-1)}, \\
& a_{r}^{(k)}=\frac{1}{\Lambda^{(k)}}\left[\frac{1}{2} b_{r 0}+\sum_{s=1}^{\infty} b_{r s} a_{s}{ }^{(k-1)}\right], \quad r \geqq 1 .
\end{aligned}
$$

If $a_{0}=0$ (as, for example, if $y$ is an odd function) or if $a_{0}$ is small compared with subsequent coefficients, some other appropriate coefficient must be taken equal to unity, and Eqs. (8) and (9) are modified in an obvious way.

In order to find further eigenvalues of the integral equation, the dominant latent root of $\left[b_{r s}\right]$ must be removed in the usual manner, as described, for example, in Modern Computing Methods [4, p. 26].

The methods described above for handling the algebraic equations are standard and straightforward. More sophisticated methods, particularly methods for accelerating the convergence of the iterative processes, may be more appropriate in certain cases; a comprehensive account of the methods available is given by Wilkinson [5].

Example 1. Consider the equation

$$
y(x)=-\cosh x+\lambda \int_{-1}^{1} \cosh (x+\xi) y(\xi) d \xi .
$$

The solution can be shown to be 


$$
y(x)=\frac{\cosh x}{\frac{1}{2} \lambda \sinh 2+(\lambda-1)} .
$$

Suppose, for example, $\lambda=2$. The solution then has the Chebyshev expansion

$$
\begin{aligned}
y(x)= & \frac{1}{2} \times 0.54727+0.05868 T_{2}(x)+0.00118 T_{4}(x) \\
& +0.00001 T_{6}(x)+\cdots .
\end{aligned}
$$

The coefficients $b_{r s}$ for the kernel cosh $(x+\xi)$ are required only for even $r$ and $s$, and are shown in Table 1; the steps in the iterative process are shown in Table 2. (A tick shows that the previous entry in a column is repeated.)

TABLE 1.

The Coefficients $b_{r s}$ for the Kernel cosh $(x+\xi)$.

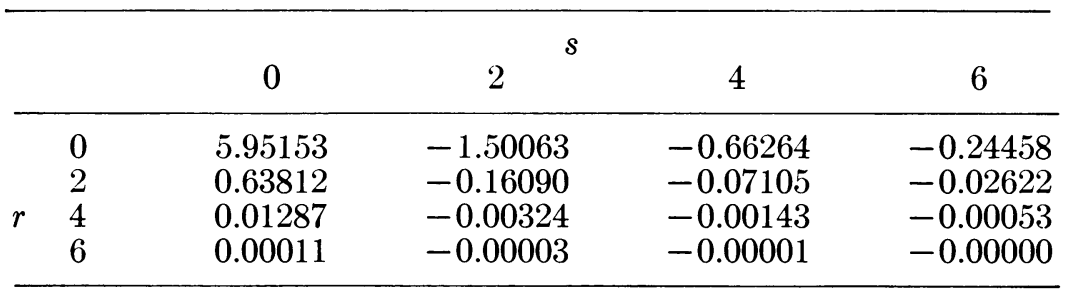

TABLE 2.

Solution of Eq. (10) with $\lambda=2$. (Example 1)

\begin{tabular}{clllc}
\hline$k$ & \multicolumn{1}{c}{$a_{0}$} & \multicolumn{1}{c}{$a_{2}$} & \multicolumn{1}{c}{$a_{4}$} & $a_{6}$ \\
\hline 1 & 0.51 & 0.04 & & \\
2 & 0.536 & 0.053 & 0.001 & \\
3 & 0.5438 & 0.0570 & 0.0011 & \\
4 & 0.54623 & 0.05819 & 0.00117 & 0.00001 \\
5 & 0.54697 & 0.05853 & 0.00118 & $\sqrt{ }$ \\
6 & 0.54718 & 0.05864 & $\sqrt{ }$ & $\sqrt{ }$ \\
7 & 0.54724 & 0.05867 & $\sqrt{ }$ & $\sqrt{ }$ \\
8 & 0.54727 & 0.05868 & $\sqrt{ }$ & $\sqrt{ }$ \\
9 & $\sqrt{ }$ & $\sqrt{ }$ & & \\
\hline
\end{tabular}

TABLE 3.

Solution of the Eigenvalue Problem (11). (Example 2)

\begin{tabular}{ccccc}
\hline$k$ & \multicolumn{1}{c}{$\Lambda$} & $a_{2}$ & $a_{4}$ & $a_{6}$ \\
\hline 1 & 2.976 & 0.107 & 0.002 & \\
2 & 2.813873 & 0.107220 & 0.002162 & 0.000018 \\
3 & 2.813431 & $\sqrt{ }$ & $\sqrt{ }$ & $\sqrt{ }$ \\
4 & $\sqrt{ }$ & & & \\
\hline
\end{tabular}

Example 2. Next consider the eigenvalue problem

$$
y(x)=\lambda \int_{-1}^{1} \cosh (x+\xi) y(\xi) d \xi .
$$


The eigenvalue corresponding to an even characteristic function can be shown to be $1 /\left(1+\frac{1}{2} \sinh 2\right)=0.355438$, and the characteristic function is a multiple of $\cosh x$. The $b_{r s}$ are the same as those of Example 1, and the steps in the iteration are set out in Table 3. The value obtained for $\lambda$ is $1 / 2.813431=0.355438$, and the characteristic function is $y(x)=\frac{1}{2}+0.107220 T_{2}(x)+0.002162 T_{4}(x)+$ $0.000018 T_{6}(x)+\cdots$, which, it may be verified, is equal to $0.394924 \cosh x$.

Convergence. The process (7) does not converge for all $\lambda$. It fails, obviously, if $\lambda=2 / b_{00}$ or if $\lambda=1 / b_{r r}$ for any $r \geqq 1$; and if $\lambda$ is one of the eigenvalues of the system no solution exists in general. If $U$ and $L$ are the matrices

$$
\left[\begin{array}{ccccc}
0 & b_{01} & b_{02} & b_{03} & \ldots \\
0 & 0 & b_{12} & b_{13} & \ldots \\
0 & 0 & 0 & b_{23} & \cdots \\
0 & 0 & 0 & 0 & \ldots \\
\ldots & \ldots & \ldots & \ldots
\end{array}\right] \text { and }\left[\begin{array}{cccccc}
\frac{1}{2} b_{00} & 0 & 0 & 0 & \ldots \\
\frac{1}{2} b_{10} & b_{11} & 0 & 0 & \ldots \\
\frac{1}{2} b_{20} & b_{21} & b_{22} & 0 & \ldots \\
\frac{1}{2} b_{30} & b_{31} & b_{32} & b_{33} & \ldots \\
\ldots \ldots & \ldots & \ldots & \ldots
\end{array}\right]
$$

respectively, the process converges if and only if all values of $\mu$ which satisfy

$$
\operatorname{det}(\mathrm{U}+\mu \mathrm{L}-\mu \Lambda \mathrm{I})=0,
$$

where $\Lambda=1 / \lambda$, are less than one in magnitude. The determinant is, of course, infinite, but only as many rows and columns as are used in the iterative process need be considered. Thus, when applied to Eq. (10), the process converges (at any rate as far as the $a_{6}$ term) if and only if all the roots of the equation

$$
\left|\begin{array}{cccc}
(2.97576-\Lambda) \mu & -1.50063 & -0.66264 & -0.24458 \\
0.31906 \mu & -(0.16090+\Lambda) \mu & -0.07105 & -0.02622 \\
0.00643 \mu & -0.00324 \mu & -(0.00143+\Lambda) \mu & -0.00053 \\
0.00005 \mu & -0.00003 \mu & -0.00001 \mu & -\Lambda \mu
\end{array}\right|=0
$$

are less than one in magnitude. If $\Lambda$ is large compared with, say, 0.006 , the offdiagonal terms in the third and fourth rows may be neglected, and the largest root is then seen to be

$$
\mu \simeq \frac{1.50 \times 0.32}{(2.98-\Lambda)(0.16+\Lambda)}
$$

It can therefore be deduced that the process converges if $\lambda$ lies within one of the approximate ranges

$$
-3.26<\lambda<0.32
$$

and

$$
0.36<\lambda \ll 170 .
$$

The restriction $\lambda \ll 170$ follows from the assumption that $\Lambda$ is large compared with 0.006 ; but further investigation using the third and fourth rows shows that this 
restriction can be dropped. Thus, in the case of Eq. (10), the process converges for all positive $\lambda$ except for a small region near the eigenvalue 0.355438 .

When $\lambda$ does not lie within the region of convergence, or when the convergence is unreasonably slow, a slight modification of the process may be used. In such cases it will be found that the diagonal term in one (or more) of the set of Eqs. (2) is small compared with one (or more) of the other terms in the same equation. Thus in the solution of (10) with $\lambda=\frac{1}{3}$ the equation corresponding to $r=0$ is

$$
0.00808 a_{0}+0.50021 a_{2}+0.22088 a_{4}+0.08153 a_{6}+\cdots=-2.53213 .
$$

The smallness of the $a_{0}$ term compared with the $a_{2}$ term causes the trouble here. The $a_{2}$ term is therefore eliminated using the equation corresponding to $r=2$, giving

$$
a_{0}+3.5793 a_{4}+1.3211 a_{6}+\cdots=-41.0323 .
$$

If, now, in process (7) the equation corresponding to $r=0$ is replaced by

$$
a_{0}{ }^{(k)}=-41.0323-3.5793 a_{4}{ }^{(k-1)}-1.3211 a_{6}{ }^{(k-1)}-\cdots,
$$

the process is found to converge quite rapidly; the steps in the iteration are shown in Table 4. In general, if in the equation of the set (2) corresponding to $r=p$ the $a_{p}$ term is small compared with the $a_{q}$ term, the latter is eliminated using the equation corresponding to $r=q$; and the resulting equation is used to replace the one corresponding to $r=p$ in (7). Sometimes more than one equation must be treated in this way, and more than one elimination may be necessary.

Turning now to the eigenvalue problem, the process defined by (8) and (9) necessarily converges unless $\left[b_{r s}\right]$ has a second latent root of equal magnitude to $\Lambda$; the rate of convergence depends on the ratio of $\Lambda$ to the next largest latent root. It may be thought more convenient to replace Eq. (9) by

$$
a_{r}{ }^{(k)}=\frac{1}{\Lambda^{(k)}-b_{r r}}\left[\frac{1}{2} b_{r 0}+\sum_{s=1}^{r-1} b_{r s} a_{s}{ }^{(k)}+\sum_{s=r+1}^{\infty} b_{r s} a_{s}{ }^{(k-1)}\right],
$$

so that $a_{r}{ }^{(k-1)}$ can be discarded when $a_{r}{ }^{(k)}$ is calculated. This change may sometimes increase the rate of convergence; on the other hand it may prevent the process from converging altogether (as, for example, if $\Lambda$ is close to one of the $b_{r r}$ ).

TABLE 4.

Solution of Eq. (10) with $\lambda=\frac{1}{3}$.

\begin{tabular}{ccccc}
\hline$k$ & $a_{0}$ & $a_{2}$ & $a_{4}$ & $a_{6}$ \\
\hline 1 & -41.03 & -4.40 & -0.09 & \\
2 & -40.7102 & -4.3650 & -0.0880 & -0.0007 \\
3 & -40.7165 & -4.3656 & $\sqrt{ }$ & $\sqrt{ }$ \\
4 & $\sqrt{ }$ & $\sqrt{ }$ & &
\end{tabular}

Discontinuous Kernels. If the kernel $K(x, \xi)$ is discontinuous or has discontinuous derivatives the above treatment is not invalidated, for $K(x, \xi)$ may still possess a Chebyshev series. This series will be slowly convergent, however, and the computing time will therefore be increased. It frequently happens that all the dis- 
continuities (or discontinuous derivatives) occur on the line $x=\xi$, and in this case the effect of the discontinuities can be removed by proceeding as follows.

First find functions $P(x, \xi), Q(x, \xi)$, both of which are continuous and have continuous derivatives throughout the region $-1 \leqq x \leqq 1,-1 \leqq \xi \leqq 1$, such that

$$
\begin{aligned}
& K(x, \xi)=P(x, \xi) \quad \text { for }-1 \leqq \xi<x \\
& =Q(x, \xi) \text { for } x<\xi \leqq 1 .
\end{aligned}
$$

The integral equation can then be written as

$$
y(x)=F(x)+\lambda \int_{-1}^{x} P(x, \xi) y(\xi) d \xi+\lambda \int_{x}^{1} Q(x, \xi) y(\xi) d \xi .
$$

By taking $Q(x, \xi)$ as zero an equation of the Volterra type is obtained, so that the work which follows is equally applicable to Fredholm or Volterra equations.

The integral Eq. (12) can be reduced to the set of algebraic Eqs. (2) by putting

$$
b_{r s}=c_{r s}+d_{r s}
$$

where $c_{r s}$ and $d_{r s}$ are defined by

$$
\begin{aligned}
& \sum_{r=0}^{\infty}{ }^{\prime} c_{r s} T_{r}(x)=\int_{-1}^{x} P(x, \xi) T_{s}(\xi) d \xi, \\
& \sum_{r=0}^{\infty} d_{r s} T_{r}(x)=\int_{x}^{1} Q(x, \xi) T_{s}(\xi) d \xi .
\end{aligned}
$$

Let $P(x, \xi), Q(x, \xi)$ have Chebyshev expansions

$$
\begin{aligned}
& P(x, \xi)=\sum_{r=0}^{\infty} \sum_{s=0}^{\infty}{ }^{\prime} p_{r s} T_{r}(x) T_{s}(\xi), \\
& Q(x, \xi)=\sum_{r=0}^{\infty} \sum_{s=0}^{\infty}{ }^{\prime} q_{r s} T_{r}(x) T_{s}(\xi),
\end{aligned}
$$

and let $\theta_{r s}, \phi_{r s}$ be defined by the formulae

$$
\begin{aligned}
\theta_{r s} & =p_{r, s-1}-p_{r, s+1} & \phi_{r s} & =q_{r, s-1}-q_{r, s+1} \\
\theta_{r 0} & =0 & \phi_{r 0} & =0 \\
\theta_{r,-s} & =-\theta_{r s} & \phi_{r,-s} & =-\phi_{r s} \\
\theta_{-r, s} & =\theta_{r s} & \phi_{-r, s} & =\phi_{r s} .
\end{aligned}
$$

It can then be shown that

$$
\begin{aligned}
c_{r s}=\frac{1}{8} \sum_{t=1}^{\infty} \frac{1}{t}\left(\theta_{r+t, s+t}-2(-1)^{t} \theta_{r, s+t}+\theta_{r-t, s+t}\right. & \\
& \left.\quad-\theta_{r+t, s-t}+2(-1)^{t} \theta_{r, s-t}-\theta_{r-t, s-t}\right)
\end{aligned}
$$

and

$$
\begin{aligned}
d_{r s}=\frac{1}{8} \sum_{t=1}^{\infty} \frac{1}{t}\left(-\phi_{r+t, s+t}+2 \phi_{r, s+t}-\right. & \phi_{r-t, s+t} \\
& \left.+\phi_{r+t, s-t}-2 \phi_{r, s-t}+\phi_{r-t, s-t}\right) .
\end{aligned}
$$


Once $c_{r s}$ and $d_{r s}$ have been obtained, the problem reduces to the solution of the set of Eqs. (2), which can be carried out as before.

TABLE 5 .

Solution of Eq. (13). (Example 3)

\begin{tabular}{cllcc}
\hline$k$ & \multicolumn{1}{c}{$a_{0}$} & \multicolumn{1}{c}{$a_{2}$} & \multicolumn{1}{c}{$a_{4}$} & $a_{6}$ \\
\hline 1 & 1.60 & 0.17 & & \\
2 & 1.6397 & 0.1757 & 0.0035 & \\
3 & 1.640903 & 0.175936 & 0.003547 & 0.000029 \\
4 & 1.640957 & 0.175944 & 0.003548 & $\sqrt{ }$ \\
5 & 1.640959 & $\sqrt{ }$ & $\sqrt{ }$ & $\sqrt{ }$ \\
6 & $\sqrt{ }$ & & & \\
\hline
\end{tabular}

Example 3. As a final example consider the equation

$$
y(x)=1-\frac{1}{2} \int_{-1}^{1} K(x, \xi) y(\xi) d \xi,
$$

where

$$
\begin{aligned}
K(x, \xi) & =1+\xi-x-x \xi, \quad-1 \leqq \xi \leqq x \\
& =1-\xi+x-x \xi, \quad x \leqq \xi \leqq 1 .
\end{aligned}
$$

The solution is clearly an even function, and it is only necessary to find $b_{r s}$ for even $r$ and $s$. The steps in the iteration are set out in Table 5 . It may be verified that the final solution agrees with the correct solution, $y(x)=\cosh x / \cosh 1$.

University of Bradford

Mathematics Department

Yorkshire, England

1. D. L. ElLiotr, "The numerical solution of integral equations using Chebyshev polynomials," J. Austral. Math. Soc., v. 1, 1959-60, pp. 344-356. MR 23 \#B1131.

2. D. L. ELLiotT "A Chebyshev series method for the numerical solution of Fredholm integral equations," Comput. J., v. 6, 1963-64, pp. 102-111. MR 27 \#5386.

3. R. E. Scraton, "The solution of linear differential equations in Chebyshev series," Comput. $J .$, v. 8, 1965, pp. 57-61. MR $32 \# 603$.

4. Modern Computing Methods, National Physical Laboratory, Notes on Applied Science no. 16, H.M.S.O., London, 1961. MR 22 \#8637.

5. J. H. WiLkinson, The Algebraic Eigenvalue Problem, Clarendon Press, Oxford, 1965. MR $32 \# 1894$. 